\title{
PRINCIPAL CONGRUENCES OF PSEUDOCOMPLEMENTED SEMILATTICES AND CONGRUENCE EXTENSION PROPERTY
}

\author{
H. P. SANKAPPANAVAR
}

\begin{abstract}
Principal congruences of pseudocomplemented semilattices are characterized and shown to be definable. This characterization is then applied to give a new proof of the fact that the variety of pseudocomplemented semilattices has the congruence extension property.
\end{abstract}

1. Introduction. In this note ${ }^{1}$ the principal congruences of pseudocomplemented semilattices are characterized. We then give, as an application of this characterization, a new (direct) proof of the fact that the variety of pseudocomplemented semilattices has the congruence extension property, a result which was first proved by Jones [8] using the corresponding result for pseudocomplemented distributive lattices of Grätzer and Lakser [7]. Moreover, our characterization shows that the variety in question has definable principal congruences in the sense of Baldwin and Berman [1], indicating how the defining formula can be explicitly written down. For an analogous characterization and the definability of principal congruences of pseudocomplemented distributive lattices see Lakser [9], and the definability of principal congruences of semilattices is implicit in Dean and Oehmke [4], while Fried and Grätzer [6] have shown that the principal congruences in the variety (of weakly associative lattices) generated by $Z$, the smallest nontransitive tournament, are definable.

2. Main result. An algebra $\left\langle S ; \wedge,{ }^{*}, 0\right\rangle$ is a pseudocomplemented semilattice (PCS) iff $\langle S ; \wedge, 0\rangle$ is a $\wedge$-semilattice with least element 0 and ${ }^{*}$ is a unary operation on $S$ such that for $a \in S, a^{*}$ is the pseudocomplement of $a$ in $S$, i.e., $x \wedge a=0$ in $S$ iff $x \leqslant a^{*}$. The class of PCS's is known to be an equational class (cf. [2]). In this paper $S$ denotes a PCS and $0^{*}$ (being the

Received by the editors March 28, 1977.

AMS (MOS) subject classifications (1970). Primary 06A20; Secondary 08A25.

Key words and phrases. Pseudocomplemented semilattice, principal congruence, definable congruence, congruence extension property.

${ }^{1}$ The work of this note is essentially a part of the author's Ph.D. Thesis, University of Waterloo, 1974 and was supported by an Ontario Graduate Fellowship. The author is grateful to his supervisor Professor Stanley Burris and also to Dr. Bulman-Fleming for their interest in this work. The final draft of this note was written in January, 1977 while the author was visiting the Department of Pure Mathematics, University of Waterloo, and was supported by NRC Grant A7256. 
greatest element) is denoted by 1 . It is well known (cf. [5]) that the set $B(S)$ of closed elements $a$ (i.e., $a=a^{* *}$ ) of $S$ is a Boolean algebra whose meet and complement operations are respectively the meet and ${ }^{*}$ in $S$ and whose join operation $\dot{V}$ is given by

$$
a \dot{\vee} b=\left(a^{*} \wedge b^{*}\right)^{*}
$$

From this one notes that an equivalence relation on $B(S)$ is a congruence (in the usual sense) iff it is a congruence when $B(S)$ is regarded as a PCS.

LEMMA 1. Let $a, b \in S$ be such that $a \leqslant b$ and $a^{*}=b^{*}$. Then the principal congruence $\theta(a, b)$ is characterized by $x \equiv y(\theta(a, b))$ iff either $x=y$, or $x \leqslant b, y \leqslant b$ and $x \wedge a=y \wedge a$. Moreover, $x \equiv y(\theta(a, b))$ implies $x^{*}=y^{*}$.

Proof. Use the fact that $a \leqslant b^{* *}$ implies $(a \wedge b)^{*}=a^{*}$ in any PCS.

For $a, b$ in $S$ let us denote by $\theta_{\text {semilat }}(a, b)$ the principal semilatticecongruence on $S$, regarding $S$ simply as a semilattice, generated by $\langle a, b\rangle$. Then it should be noted from Lemma 1 and a result in Dean and Oehmke [4] that if $a, b$ are as in Lemma 1 then $\theta_{\text {semilat }}(a, b)$ is indeed a (PCS-) congruence on $S$.

For $a \in S$, define a relation $\hat{a}$ on $S$ as follows:

$$
\langle x, y\rangle \in \hat{a} \quad \text { iff } x \wedge a=y \wedge a, \quad x, y \in S .
$$

Then using the identity $x \wedge(x \wedge y)^{*}=x \wedge y^{*}$ true in any PCS one readily sees that $\hat{a}$ is a congruence on $S$; moreover it is easily observed from Dean and Oehmke that $\theta(a, 1)=\hat{a}=\theta_{\text {semilat }}(a, 1)$.

LEMMA 2. Let $t \in S$ and $\psi \in \operatorname{Con} S$, the congruence lattice of $S$. Then $\hat{t} \vee \psi=\hat{t} \circ \psi \circ \hat{t}$ (where $\circ$ denotes relative product).

Proof. It suffices to prove $\psi \circ \hat{t} \circ \psi \subseteq \hat{t} \circ \psi \circ \hat{t}$. Let $x \psi x_{1} \hat{t} x_{2} \psi y$. Since $x_{1} \wedge t=x_{2} \wedge t$, we get $x \hat{t} x \wedge t \psi x_{1} \wedge t=x_{2} \wedge t \psi y \wedge t \hat{t} y$.

We are now ready to give the main result characterizing the principal congruences.

TheOREM 1. Let $a, b \in S$. Then

$$
\begin{aligned}
\theta(a, b)= & \theta_{\text {semilat }}\left(\left(a^{*} \wedge b\right)^{*} \wedge\left(a \wedge b^{*}\right)^{*}, 1\right) \\
& \circ \theta_{\text {semilat }}\left(a \wedge b^{* *}, a^{* *} \wedge b\right) \\
& \circ \theta_{\text {semilat }}\left(\left(a^{*} \wedge b\right)^{*} \wedge\left(a \wedge b^{*}\right)^{*}, 1\right) .
\end{aligned}
$$

Proof. We know $\left\langle a^{*}, b^{*}\right\rangle \in \theta(a, b)$, so $\left\langle a^{*} \wedge b, 0\right\rangle \in \theta(a, b)$ which implies $\left\langle\left(a^{*} \wedge b\right)^{*}, 1\right\rangle \in \theta(a, b)$ and by symmetry, $\left\langle\left(a \wedge b^{*}\right)^{*}, 1\right\rangle \in \theta(a, b)$, hence $\left\langle\left(a^{*} \wedge b\right)^{*} \wedge\left(a \wedge b^{*}\right)^{*}, 1\right\rangle \in \theta(a, b)$, thus we get $\theta_{\text {semilat }}\left(\left(a^{*} \wedge b\right)^{*} \wedge\right.$ $\left.\left(a \wedge b^{*}\right)^{*}, 1\right) \subseteq \theta(a, b)$. Also we have $\left\langle a \wedge b^{* *}, b\right\rangle \in \theta(a, b)$ and $\left\langle a, a^{* *} \wedge\right.$ $b\rangle \in \theta(a, b)$, so $\left\langle a \wedge b^{* *}, a^{* *} \wedge b\right\rangle \in \theta(a, b)$ which gives that $\theta_{\text {semilat }}(a \wedge$ $\left.b^{* *}, a^{* *} \wedge b\right) \subseteq \theta(a, b)$.

Thus, to complete the proof we only need to show that $\langle a, b\rangle$ belongs to the expression on the right side of the equation. Now it is straightforward to 
verify that

$$
\begin{aligned}
a^{* *} \wedge\left[\left(a^{*} \wedge b^{* *}\right)^{*} \wedge\left(a^{* *} \wedge b^{*}\right)^{*}\right] \\
=b^{* *} \wedge\left[\left(a^{*} \wedge b^{* *}\right)^{*} \wedge\left(a^{* *} \wedge b^{*}\right)^{*}\right]
\end{aligned}
$$

Hence from the observation immediately preceding Lemma 2 it follows that $\left\langle a^{* *}, b^{* *}\right\rangle$ is in $\theta_{\text {semilat }}\left(\left(a^{*} \wedge b^{*}\right)^{*} \wedge\left(a \wedge b^{*}\right)^{*}, 1\right)$ which implies that $\langle a, a$ $\left.\wedge b^{* *}\right\rangle$ and $\left\langle a^{* *} \wedge b, b\right\rangle$ are also in this congruence. Then it is immediate that $\langle a, b\rangle$ belongs to the expression on the right of the equation. This completes the proof.

Lemma 3. Let $a, b \in S$ with $a^{*}=b^{*}$. Then

$$
\begin{aligned}
\theta(a, b)= & (\theta(a, a \wedge b) \circ \theta(a \wedge b, b)) \\
& \cup(\theta(a \wedge b, b) \circ \theta(a, a \wedge b)) .
\end{aligned}
$$

Proof. Let $\psi_{1}=\theta(a, a \wedge b)$ and $\psi_{2}=\theta(a \wedge b, b)$; then it suffices to show that $\left(\psi_{1} \circ \psi_{2}\right) \cup\left(\psi_{2} \circ \psi_{1}\right)$ is transitive. Let us first suppose that $\langle x, y\rangle \in$ $\psi_{1} \circ \psi_{2}$ and $\langle y, z\rangle \in \psi_{1} \circ \psi_{2}$. Then $x \psi_{1} t \psi_{2} y \psi_{1} u \psi_{2} z$ for some $t, u$ in $S$. From this one easily checks using Lemma 1 that $x \wedge b=z \wedge a$, and hence $x \psi_{1} x \wedge b$ and $z \wedge a \psi_{2} z$, implying $x \psi_{1} \circ \psi_{2} y$. Next let $\langle x, y\rangle \in \psi_{1} \circ \psi_{2}$ and $\langle y, z\rangle \in \psi_{2} \circ \psi_{1}$. Then for some $t, u \in S$ we have $x \psi_{1} t \psi_{2} y \psi_{2} u \psi_{1} z$ and so $x \psi_{1} t \psi_{2} u \psi_{1} z$. Applying Lemma 1 one sees that $t=u$ and hence $x \psi_{1} z$. The other cases being similar, the proof is complete.

From Lemmas 1 and 3 it follows immediately that $\theta(a, b)$ with $a^{*}=b^{*}$ is definable while it is clear that $\hat{t}$ is definable and hence from Theorem 1 we conclude immediately the following.

COROLlARY. The variety of pseudocomplemented semilattices has definable principal congruences.

Proof. By looking at Lemmas 1, 2 and 3 and Theorem 1 the reader can easily write down the formula which defines a principal congruence.

3. Application. Recall that a class of algebras $K$ has the congruence extension property (CEP) iff given $A, B \in K, A$ a subalgebra of $B$, then every congruence $\theta$ on $A$ is the restriction of some congruence on $A$.

Jones [8] has proved that the variety PCS of pseudocomplemented semilattices has CEP. In his proof he associates with each PCS a pseudocomplemented distributive lattice in a suitable way and then uses a result of Grätzer and Lakser [7] which says that the class of distributive pseudocomplemented lattices has CEP. Since a PCS has already a semilattice structure built into it and the semilattices are known to have CEP, one is naturally led to ask whether it is possible to use this property of semilattices to prove Jones' result, instead of going through distributive pseudocomplemented lattices. We now give, as an application of Theorem 1, a positive answer to this question. For this purpose we need the following lemma. 
LEMMA 4. Let $S_{1}$ be a PCS-subalgebra of $S, t \in S_{1}$, and let $\alpha \in$ Con $S$. Then

$$
\left.(\hat{t} \vee \alpha)\right|_{s_{1}}=\left.\left.(\hat{t})\right|_{s_{1}} \vee(\alpha)\right|_{s_{1}}=\left.\left.\left.(\hat{t})\right|_{s_{1}} \circ(\alpha)\right|_{s_{1}} \circ(\hat{t})\right|_{s_{1}}
$$

Proof. It is obvious that the expression on the extreme right is contained in the one on the left. Let $\left.\langle x, y\rangle \in(\hat{t} \vee \alpha)\right|_{s_{1}}$. Since $\langle x, y\rangle \in \hat{t} \vee \alpha$, there exist elements $x=x_{0}, x_{1}, \ldots, x_{n}=y$ in $S$ such that

$$
x=x_{0} \hat{t} x_{1} \alpha x_{2} \hat{t} x_{3} \alpha \cdots \alpha x_{n}=y \text {. }
$$

Then we get

$$
x \wedge t=x_{1} \wedge t \alpha x_{2} \wedge t=x_{3} \wedge t \alpha \cdots \alpha y \wedge t .
$$

From this it follows that $\langle x \wedge t, y \wedge t\rangle \in \alpha$. Since $x, y, t \in S_{1}$, we thus have $\left.\langle x \wedge t, y \wedge t\rangle \in(\alpha)\right|_{s_{1}}$. Also we know $\langle x, x \wedge t\rangle \in \hat{t}$ and $\langle y \wedge t, y\rangle \in \hat{t}$ and hence $\left.\langle x, x \wedge t\rangle \in(\hat{t})\right|_{s_{1}}$ and $\left.\langle y \wedge t, y\rangle \in(\hat{t})\right|_{s_{1}}$. Then it immediately follows that $\left.\left.\left.\langle x, y\rangle \in(\hat{t})\right|_{s_{1}} \circ(\alpha)\right|_{s_{1}} \circ(\hat{t})\right|_{s_{1}}$, proving the lemma.

Theorem 2. The class PCS has CEP.

Proof. Let $S_{1}$ be a PCS-subalgebra of $S$ and let $a, b \in S_{1}$. Suppose $\theta^{s_{1}}(a, b)$ denotes the principal congruence on $S_{1}$ generated by $\langle a, b\rangle$ and $\theta^{S}(a, b)$ the principal congruence on $S$ generated by $\langle a, b\rangle$. Then by Theorem 1 we have

$$
\begin{aligned}
\theta^{s}(a, b)= & \theta_{\text {semilat } s}\left(\left(a^{*} \wedge b\right)^{*} \wedge\left(a \wedge b^{*}\right)^{*}, 1\right) \\
& \vee \theta_{\text {semilat } s}\left(a \wedge b^{* *}, a^{* *} \wedge b\right)
\end{aligned}
$$

Hence by Lemma 4 we get

$$
\begin{aligned}
\left.\theta^{s}(a, b)\right|_{s_{1}}= & \left.\left(\theta_{\text {semilat } s}\left(\left(a^{*} \wedge b\right)^{*} \wedge\left(a \wedge b^{*}\right)^{*}, 1\right)\right)\right|_{s_{1}} \\
& \left.\vee\left(\theta_{\text {semilat } s}\left(a \wedge b^{* *}, a^{* *} \wedge b\right)\right)\right|_{s_{1}} .
\end{aligned}
$$

Using the CEP of semilattices we obtain

$$
\begin{aligned}
&\left(\theta_{\text {semilat } s}\right.\left.\left(\left(a^{*} \wedge b\right)^{*} \wedge\left(a \wedge b^{*}\right)^{*}, 1\right)\right)\left.\right|_{s_{1}} \\
&=\theta_{\text {semilat } S_{1}}\left(\left(a^{*} \wedge b\right)^{*} \wedge\left(a \wedge b^{*}\right)^{*}, 1\right)
\end{aligned}
$$

and

$$
\left.\left(\theta_{\text {semilat } S}\left(a \wedge b^{* *}, a^{* *} \wedge b\right)\right)\right|_{S_{1}}=\theta_{\text {semilat } S_{1}}\left(a \wedge b^{* *}, a^{* *} \wedge b\right) .
$$

Thus we have

$$
\begin{aligned}
\left.\theta^{s}(a, b)\right|_{S_{1}}= & \theta_{\text {semilat } s_{1}}\left(\left(a^{*} \wedge b\right)^{*} \wedge\left(a \wedge b^{*}\right), 1\right) \\
& \vee \theta_{\text {semilat } s_{1}}\left(a \wedge b^{* *}, a^{* *} \wedge b\right)
\end{aligned}
$$

From this it follows using Theorem 1 again that $\left.\theta^{s}(a, b)\right|_{s_{1}}=\theta^{s_{1}}(a, b)$. Then by a theorem in Day [3] we see that the class PCS has CEP, proving the theorem. 
Concluding remarks. Baldwin and Berman [1] showed that if a variety is locally finite and has CEP then it has definable congruences. Jones [8] has proved that the variety PCS is locally finite. Accepting the proof of Jones [8] of the fact that PCS has CEP, it then follows from the above result of Baldwin and Berman that the PCS has definable congruences. (Theorem 1 was proved before the Baldwin-Berman result.) It is indeed interesting to compare this with our present work wherein we first showed that PCS has definable congruences by giving an explicit formula (without using CEP!) and then we used it to show that PCS has CEP.

It would be interesting to see if the CEP of pseudocomplemented distributive lattice can be deduced (we suspect so) from that of pseudocomplemented semilattices.

\section{REFERENCES}

1. J. Baldwin and J. T. Berman, The number of subdirectly irreducible algebras in a variety, Algebra Universalis 5 (1975), 379-389.

2. R. Balbes and A. Horn, Stone lattices, Duke Math. J. 37 (1970), 537-546.

3. A. Day, A note on the congruence extension property, Algebra Universalis 1 (1971), 234-235.

4. R. A. Dean and R. H. Oehmke, Idempotent semigroups with distributive right congruence lattices, Pacific J. Math. 14 (1964), 1187-1209.

5. O. Frink, Pseudocomplements in semilattices, Duke Math. J. 29 (1962), 505-514.

6. E. Fried and G. Grätzer, A non-associative extension of the class of distributive lattices, Pacific J. Math. 49 (1973), 59-78.

7. G. Grätzer and H. Lakser, The structure of pseudocomplemented distributive lattices. II. Congruence extension and amalgamation, Trans. Amer. Math. Soc. 156 (1971), 343-358.

8. J. T. Jones, Pseudocomplemented semilattices, Ph.D. Thesis, Univ. of California, Los Angeles, 1972.

9. H. Lakser, Principal congruences of pseudocomplemented distributive lattices, Proc. Amer. Math. Soc. 37 (1973), 32-36.

10. H. P. Sankappanavar, A study of congruence lattices of pseudocomplemented semilattices, Ph.D. Thesis, Univ. of Waterloo, 1974.

Instituto de Matematica, Universidade Federal de Bahia, Salvador, Bahia 40.000, BRASIL

Department of Pure Mathematics, University of Waterloo, Waterloo, Ontario, Canada 